\title{
Educación de educadores: caso Gimnasio Vermont
}

\author{
Education of educators: Gimnasio Vermont case
}

Alfonso Suárez Gómez, María V. Quijano, Raúl González, Nubia de Parra, María Haydée Becerra,

Grupo de Epistemología de la Facultad de Ciencias (GEC). Pontificia Universidad Javeriana. asuarez@javeriana.edu.co

\section{Resumen}

Se reportan los resultados del trabajo de investigación docente del GEC, (Grupo de Epistemología de las Ciencias) inicialmente nombrado como (GEFC) (grupo interdisciplinario de epistemología de las ciencias), que se gestó hacia 1984, a raíz de la preocupación de unos pocos docentes de Física y Matemática, acerca de los problemas de la enseñanza, del aprendizaje, y de la incidencia de involucrar el conocimiento de la historia y la epistemología en tales procesos.

Como resultado de este trabajo, el grupo no sólo ha realizado cursos de capacitación para docentes, tanto de la educación básica primaria y seaundaria como del nivel universitario de estas áreas, tendiente a resolver el profundo abismo que normalmente ha habido para los estudiantes entre el colegio y la universidad, sino que este grupo académico creó y puso en funcionamiento el programa de postgrado "Especialización en Docencia de las Ciencias Naturales y las Matemáticas desde la Perspectiva de la construcción del conocimiento". Este programa ya cuenta con 17 promociones, 187 graduados; un número importante de ellos (5\%) enviados por las directivas del Gimnasio Vermont. Uno de los efectos fundamentales del programa en esta institución se ha visto reflejado en las pruebas de Estado (ICFES), en las auales ocupaba el lugar 126 entre los mejores colegios de Colombia en el año 2000, alcanzando en el 2008 el primer lugar, y en el 2009 el segundo lugar a nivel nacional .

Palabras clave

Educación, Educadores, Constructivismo, historia, epistemología, Gimnasio Vermont.

\section{Abstract}

We report part of the results of teaching research of the Sciences Epistemology Group (GEC), that involves the history and Epistemology in the teaching and learning process of Sciences.

This line of research takes us to the need to reformulate teacher's and students work, in order to give the students the opportunity to face their academic work as a constructive process driven to solve the deep gap between school an the university. To reach this goal, the GEC develop their own theories, and probes that in the practice, through the "Especialización en Docencia de las Ciencias Naturales y las Matemáticas desde la Perspectiva de la construcción del conocimiento"

In this paper we use the case of Gimnasio Vermont as an indicator of the measure of our successful of this work, due to this school was in the 126 place in the dassification of schools measured for the ICFES in the $\mathbf{2 0 0 0}$ year and in the $\mathbf{2 0 0 8}$ reach the first place to the national level.

Keywords

Education, educators, constructive process, history, epistemology, Gimnasio Vermont. 


\section{Introducción}

La sociedad moderna considera a la Educación como la actividad estratégica, a los estudiantes y docentes como el reaurso humano dave, y a las Ciencias Naturales y las Matemáticas, como las disciplinas del saber que permitirán preparar mejor a los educandos para lograr un rápido proceso de cambio, tanto en lo humano como en lo tecnológico, que posibilite un desarrollo económico, social y cultural armónico. Es obvio que no existirá una verdadera democracia, hasta que el acceso a una educación que fomente una adecuada racionalidad, no sea una realidad para toda la población.

La solución del problema sólo se podrá alcanzar con una visión dara de la totalidad y la acción consecuente sobre ésta. Una de las misiones más importantes de la educación será la de contribuir a que en cada individuo se construyan, tanto la visión como la acción mencionadas, ya que con ellas cada individuo opera sobre el grupo, retroalimentando a los demás y siendo retroalimentado por ellos. Es importante que esta interacción sea encauzada hacia una acción constructiva y hacia el bienestar personal de cada individuo, estrechamente ligado al bienestar de la comunidad, conformada por todos y cada uno de sus elementos.

Es así como desde nuestras dases para Ingenieros, Licenciados y estudiantes de otras carreras hemos venido explorando el problema básico en pedagogía aual es el "formar individuos aptos para la invención y capaces de impulsar el progreso de la sociedad del mañana." (1).

Entre las hipótesis iniciales del grupo estaba la de poner a prueba las teorías del G.E.C. (como espacio de experimentación) sobre los problemas de la enseñanza y el aprendizaje con la totalidad de los docentes de una institución educativa, independientemente de su disciplina de formación. Dado que no fue posible aplicar esta prueba, se escogió al Gimnasio Vermont como referente para este trabajo, dado que es lo más cercano a esa posibilidad y a que ha sido una política de sus directivas aportar a la formación pedagógico-didáctica de sus docentes la propuesta planteada por el grupo de investigación.

En el presente artículo ilustramos la incidencia del planteamiento teórico-práctico del GEC en un caso como el del Gimnasio Vermont, cuyas directivas han elegido 9 docentes durante los últimos 16 años, para que cursen la especialización en Docencia de las Ciencias Naturales y las Matemáticas y ayyo éxito queremos resaltar sin atribuimos más méritos que los que ellos mismos han destacado, excediéndose en buena parte de los casos en generosidad para con nosotros.

\section{Marco Teórico}

Según Piaget, en 1935 el problema fundamental en pedagogía era el de formar individuos aptos para la invención y capaces de impulsar el progreso de la sociedad del mañana. Conduía el mismo autor que para el logro de dicho objetivo, una educación del descubrimiento activo de lo verdadero, era superior a una educación que sólo consistiera en amaestrar a los individuos para que deseen de acuerdo con una voluntad ajena y para que aprendan de acuerdo con verdades simplemente aceptadas.

Hoy, 74 años después, el problema parece no haber cambiado ostensiblemente, y también hoy nos corresponde espantarnos, como lo hacía Piaget en 1965 ante la desproporción que aún subsiste entre la inmensidad de los esfuerzos realizados y la ausencia de una fundamental renovación de los métodos, de los programas, de la situación misma de los problemas y, en definitiva, de la pedagogía en su conjunto como disciplina rectora. 
Los sistemas educativos no han evolucionado con la misma velocidad que otros sectores de la actividad humana; existe descenso en la calidad de la educación, profusión de matrículas universitarias y aumento de la cifra de desempleados profesionales, siendo evidente una crisis creciente. El sistema educativo no prepara a los individuos para enfrentar los cambios acelerados que se producen en todos los órdenes: personal, social, ético, político, económico, técnico y científico.

Fue en este sentido que hacia el año 1984, un grupo de docentes de física, química, biología y matemáticas bastante inquietos y con nuevas iniciativas, iniciamos un proceso de investigación mancomunado, tendiente a buscar salidas para los problemas de la enseñanza de las ciencias naturales y las matemáticas en todos los niveles educativos, enfatizando en la búsqueda de un puente para el abismo que ha existido entre la educación media y la superior.

Los resultados de este trabajo han sido prometedores en el ámbito de los procesos de enseñanza y de aprendizaje, en los auales se conciben los conocimientos como un medio para desarrollar las diferentes potencialidades de los estudiantes y no como un fin en si mismos. También nos permitieron desarrollar en los diferentes niveles de enseñanza de las disciplinas, desde métodos alternativos para resolver problemas en cada una de ellas, hasta maneras de contribuir a la solución de problemas concretos de la sociedad, como por ejemplo: ambientales y de servicios públicos. Este enfoque nos ha permitido no sólo una mayor eficiencia en el cumplimiento de nuestra labor docente, sino que también nos ha brindado vías de solución a los problemas de formación integral tanto del estudiante como del ciudadano, más acordes con los requerimientos de la sociedad.

\section{Premisas que guiaron nuestro trabajo}

Las premisas básicas que guiaron nuestro quehacer académico se agruparon en dos ítems, que son: 1) Todos los procesos humanos y en particular los procesos educativos tienen como único fin, la realización del individuo 2) Los conocimientos: no son entes estáticos para ser aprendidos, sino instancias de formación de los alumnos; requieren de un desarrollo cognitivo para su comprensión, el cual se debe lograr durante los procesos de construcción del mismo; son peldaños para nuevas conquistas.

\section{Instrumentos utilizados en la implementación de este tipo de proceso Académico}

La epistemología y la historia nos sirvieron como base en el intento de develar nuestra realidad educativa y buscar su sentido. No sólo nos iluminaron con los conceptos más fructíferos de las tradiciones filosóficas, sino que nos suministraron datos concretos de nuestra realidad educativa y cientúfica, sirviéndonos tanto en nuestro trabajo como en la reflexión acerca del mismo, como elementos organizadores y orientadores. Nos pusieron de frente con los conceptos (tanto concientes como inconcientes) que dominaban nuestra realidad educativa, y nos sirvieron para ir estableciendo un orden e interrelación conceptual, que la iban haciendo más inteligible. En síntesis, acudimos a 1) La epistemología de las ciencias, para ayudarnos a organizar los contenidos en una progresiva secuencia de niveles de abstracción, dentro de cada unidad didáctica, así como para tratar de entender los procesos seguidos. 2) La historia nos sirvió como un hilo conductor, tanto en la dirección de los procesos, como en la búsqueda de los obstáculos con miras a removerlos. 


\section{Estrategias Utilizadas}

\section{Estrategias en el manejo del conocimiento}

Considerando el proceso de aproximación al conocimiento como un proceso social eminentemente constructivo, hemos buscado en la historia, la epistemología y nuestra propia experiencia, aquellos factores que han determinado la movilización de la cultura científica, determinando en buena parte su progreso. Como resultado de esta búsqueda, hemos hallado dos factores daves en la evolución de nuestra cultura, a saber:

1) La revisión permanente de los conceptos, que nos ha conducido a la evolución de los mismos, así como de nuestros hábitos mentales y de nuestras formas de percepción y representación.

2) El quehacer científico, que nos ha llevado a niveles de abstracción cada vez mayores, recusando nuestros sentidos, en la medida que los aparta de sus expresiones primarias. Basándonos en lo anterior, y ya en el caso de nuestras dases, las hemos intentado abocar como un proceso permanente de revisión reflexiva de los conocimientos y experiencias del estudiante, en el cual ellos aprenderán a redimensionarlos permanentemente, a la vez que van dándose cuenta de la necesidad de depuración, tanto de sus hábitos mentales, como de los hábitos de aproximación al conocimiento, reemplazando así el saber estático por uno abierto y dinámico. Esto también se intentó hacer válido para nuestro propio proceso.

3) Ya que todo el proceso anterior -al igual que todo proceso humano- esta mediado por errores, fallas, desaciertos, aproximaciones, o como quiera llamárseles. Se enfatizó a los estudiantes la importancia de aprender a interactuar con éstos en una dimensión diferente de la tradicional, logrando induso la capitalización de los mismos, al punto de convertirlos en una fuente de comprensión.

Estrategias en el caso específico en el rediseño de las actividades educativas para ejercitar al estudiante en su papel de agente transformador de la sociedad.

Hemos tenido que trascender el esquema tradicional de la dase y plantear el programa a cumplir, como parte de un trabajo más amplio, en el cual tanto el estudiante como el docente, no sólo den significado a su labor, sino que vayan respondiendo en la medida de sus posibilidades a los requerimientos de la sociedad.

En nuestro caso, como ya lo dijimos, las ciencias y las matemáticas no sólo fueron el gimnasio de la racionalidad, sino también un medio para desarrollar las potencialidades de los estudiantes y mejorar su aproximación a la naturaleza. Sus leyes, que muchas veces recusan su sentido común, contribuyen positivamente en su proceso de descentración. Las aplicaciones con beneficio propio y social no sólo le hacen partícipe, sino que lo proyectan a la comunidad y dan sentido al aprendizaje. Los procesos de construcción del conocimiento le permiten aprender acerca de los caminos que sigue la mente, al estar en contacto con los obstáculos, requerimientos y soluciones inherentes a los procesos constructivos.

Estrategias especificas para el docente

Teniendo en cuenta las consideraciones anteriores, el principal trabajo del docente no será el de "dictar dases" sino el de problematizar las concepciones y "teońas" del estudiante; es decir, el docente no sólo problematizará la "ciencia" propia del estudiante, sino los procesos que éste sigue en la búsqueda del conocimiento y la calidad y cantidad de fundamentación que tiene para el mismo. 
Convertir las aulas de clase, no sólo en instancias de investigación acerca de los problemas y procesos cognitivos, sino en lugares donde se hagan diagnósticos acerca de los problemas que enfrenta nuestra sociedad y se generen y lideren las acciones conducentes a probar la bondad de las soluciones ofrecidas.

\section{Logros}

\section{Logros de carácter general.}

Los primeros beneficiados fuimos nosotros mismos, pues al ver el proceso de enseñanza y de aprendizaje como problemas abiertos, novedosos, objetos de búsqueda y siempre llenos de sorpresas, no sólo impidió que nuestro interés por el mismo decayera con el transcurso del tiempo, sino que se constituyeron en una fuente inagotable de satisfacciones, al enfrentar con relativo éxito uno de los problemas más importantes para el ser humano, cual es el problema de cómo aprendemos.

Hemos observado que al asumir el proceso de aprendizaje por parte del estudiante como verdaderamente se presenta, es deair, como un problema abierto, mejora su aprendizaje, puesto que lo libera de estereotipos tales como que aprender equivale a memorizar, y le deja en libertad de descubrir cómo aprovechar en forma óptima sus mecanismos cognitivos.

Vimos que: a) Si queremos que los estudiantes aprendan una disciplina, por ejemplo física, deberemos darle en cuanto sea posible la oportunidad de que haga física o algo similar. b) $\mathrm{Si}$ aspiramos a que aprenda a abordar los problemas como lo haría un científico, deberemos darle la oportunidad (en lo posible), de que asuma su propia exploración alrededor del conocimiento, con todos los riesgos que esto entrañe.

Logros de carácter específico (rediseño de las actividades educativas para ejercitar al estudiante en su papel de agente transformador de la sociedad) (5)

Todo lo anterior nos ha permitido ver que quizás uno de los principales logros de un proceso educativo pueda ser el continuo mejoramiento de la racionalidad, entendida esta como coherencia entre pensamiento y acción, los cuales no sólo son perfectibles sino que si se siguen los procesos adecuados, se encuentran en continua descentración.

\section{Caso del Gimnasio Vermont}

En aras de evaluar los efectos del trabajo del GEC y en particular de la Especialización en Docencia, hemos recurrido para tal fin como primera herramienta a nuestros egresados vía procesos de acreditación, en los cuales la evaluación por parte de los especialistas ha sido muy favorable (187). Sin embargo, en nuestra pretensión de buscar valoraciones menos amigables y quizá cargadas de afecto, le hemos hecho un seguimiento a los resultados de las pruebas de Estado, ICFES (aunque, como lo tratamos de ilustrar anteriormente la intencionalidad es la formación integral y no adiestramiento para las pruebas mencionadas). Es en este sentido que consideramos los resultados del Gimnasio Vermont en estas pruebas como un indicador extemo de evaluación del proceso.

Resultados obtenidos por el colegio durante el periodo 2000-2008 (es el promedio de las ocho áreas que se evalúan): 
Tecné, Episteme y Didaxis: TE $\Delta$ No. Extraordinario, 2009

$4^{\circ}$ Congreso Internacional sobre Formación de Profesores de Ciencias

Ponencias

\begin{tabular}{|c|c|c|c|c|c|c|c|c|c|}
\hline 2000 & 2001 & 2002 & 2003 & $\begin{array}{l}2004 \\
\text { lugar }\end{array}$ & $5^{\circ}$ & 2005 & 2006 & 2007 & 2008 1er lugar \\
\hline 53,68 & 53,80 & 52,90 & 54,24 & $\begin{array}{l}60,66 \\
\text { nacional }\end{array}$ & a nivel & 59,73 & 61,66 & 59,01 & $\begin{array}{l}66,45 \\
\text { nacional }\end{array}$ a nivel \\
\hline
\end{tabular}

Una prueba fehaciente de la labor transformadora de nuestra especialización ha sido el caso del Gimnasio Vermont, en donde no sólo las apreciaciones cualitativas han mostrado un evidente progreso en los procesos de enseñanza y de aprendizaje, también el reconocimiento que varias prestigiosas universidades de Bogotá hacen de las egresadas del colegio, por su capacidad de interpretación, análisis, argumentación y habilidad para resolver problemas nuevos, en donde es necesario ir más allá de la simple repetición de procesos mecánicos y utilizar la creatividad, la racionalidad y un sentido correcto y coherente de la realidad.

Si lo anterior no fuese suficiente, están las pruebas objetivas de carácter cuantitativo, que consolidan al colegio como el mejor del país en la dasificación del ICFES, ocupando el primer lugar en las áreas de Biología y Filosofía, segundo en Química y tercero en Matemáticas, áreas sobre las auales hace mayor énfasis nuestro programa de postgrado y de aryos docentes varios cursaron la Especialización en Docencia de las Ciencias Naturales y las Matemáticas desde la perspectiva de la construcción del Conocimiento.

\section{Implicaciones metodológicas de la especialización desarrolladas en el colegio Vermont}

- Se ha incursionado en una vía de solución a algunos problemas graves del proceso educativo, como es su falta de significado. Esta introduce enormes dificultades que obstaculizan considerablemente su desarrollo.

- Bajo las condiciones anotadas, se da un desenvolvimiento más libre de tensiones, y el aprendizaje en sí brinda satisfacción a los protagonistas, tanto con los procesos como con los resultados, lo cual predispone al estudiante hacia la búsqueda permanente; es decir, hacia una actitud investigativa.

- Se ha podido demostrar que el tiempo invertido en la construcción del conocimiento no es perdido, a pesar de que al principio el avance de los estudiantes parece ser muy lento, pero una vez sentadas las bases, el avance es bastante más rápido que en el método tradicional, además de todas las otras actitudes que adquiere el estudiante, que le son vedadas por el mismo.

Los estudiantes: 1) se sienten no sólo protagonistas de sus procesos de aprendizaje, sino motor de cambios concretos en su núdeo familiar y social, además de agentes de transformación de la sociedad. 2) Encuentran sentido a los conocimientos que van adquiriendo, desde el mismo momento en que inician el proceso de aprendizaje, al aproximarse a la ciencia como a un proceso que induce actitudes objetivantes, no sólo en la dase, sino también en la vida cotidiana, sirviéndole además de modelador de sus dotes intelectuales. 3) Convierten los procesos de aprendizaje, en procesos activos y autodirigidos, que posibilitan la aproximación a una actitud de descubrimiento permanente. 4) Los estudiantes mejoraron tanto la rapidez del aprendizaje como su calidad. A este respecto debemos anotar que los y las jóvenes que parecían tener mayores dificultades al comienzo, siguieron con interés el curso, llegando a formar parte del grupo que más lo aprovechó. 


\section{Conclusiones}

Una de las formas más importantes que la sociedad ha intentado enseñar, es la forma de aproximarse al conocimiento, dada la importancia de éste, que es quizá uno de los factores que más cambios produce en una sociedad, no sólo en las formas de ver, pensar y actuar, sino que con éstas influye tanto en las nuevas prioridades de la sociedad, como en sus posibilidades para generar nuevo conocimiento $y$, en definitiva, en sus posibilidades de bienestar.

No hay sociedad que actualmente no dé un gran énfasis al valor del conocimiento, pero quizás dada la importancia de su posesión, la gran mayoría de las sociedades han priorizado su adquisición sin tener en cuenta suficientemente los efectos sociológicos debidos al proceso seguido para tal efecto.

En una sociedad mundial en la cual lo único que no cambia es el cambio, es posible afirmar que se han hecho avances significativos en un proceso de formación de formadores, buscando que éstos, desde su vivencia (en la especialización) formen a su vez, no para la "competencia en la ley de la jungla", sino para la cooperación (evidenciado en los estudiantes del Gimnasio Vermont) aspectos no fácilmente cuantificables en las "pruebas objetivas" y que según nuestro parecer y en el de los directivos de los colegios que se han sumado a esta inidativa, aportan a la calidad educativa en el sentido holístico del concepto.

\section{Bibliografía}

Piaget, J. (1970). Educación e instrucción, Buenos Aires, Ed. Proteo

Suárez, A. (1991). ¿Es posible convertir el aula de dase en una instancia para la investigación? Rev. Fac. De Ciencias U. Javeriana.Bogotá V. I, No 4, 117-139.

Vasco C E. y Suárez Gómez A. (1999). Diálogos sobre los grandes problemas del ser humano: Las estucturas mentales. Santafé de Bogotá: Cooperativa Editorial Magisterio.

Vasco, C. E.; Barrera de Aragón, M. Quijano de Castellanos, M. V. Suárez Gómez, A.; Amat, G. D. (2000). Constuyendo una propuesta pedagógica práctico-téorica: Crónicas de una experiencia. Santafé de Bogotá, D. C.: CEJA.

Vasco C E, Suárez Gómez A, Barrera de Aragón, M; Becerra Nivia, M Hay; Perdomo de Parra, N. (2004). De la teoría a la práctica en la formación de maestros en ciencias y matemáticas en Colombia. Bogotá: Editorial Pontificia Universidad Javeriana, 